\title{
Hypopituitarism due to lymphocytic hypophysitis in a patient with retroperitoneal fibrosis
}

\author{
A Alvarez, F Cordido, F Sacristán
}

\begin{abstract}
Summary
We present a 78-year-old woman with clinical acute hypopituitarism in whom pathologic findings showed lymphocytic hypophysitis and retroperitoneal fibrosis. Lymphocytic hypophysitis should be included in the differential diagnosis of hypopituitarism at any age. The association with idiopathic retroperitoneal fibrosis suggest an autoimmune origin for this entity.
\end{abstract}

Keywords: hypopituitarism, lymphocytic hypophysitis, retroperitoneal fibrosis

Hypopituitarism is most frequently due to a pituitary tumour, other common causes being surgery, pituitary radiation therapy and pituitary apoplexy. Less common causes of pituitary insufficiency include empty sella syndrome, head trauma, internal carotid artery aneurysm and infiltrative diseases that affect the hypothalamus or pituitary. ${ }^{1}$

Lymphocytic hypophysitis is being increasingly recognised as a cause of hypopituitarism, usually presenting in women during late pregnancy or the postpartum period. It is characterised by lymphocytic infiltration and destruction of the anterior pituitary gland. Depending on the stage of the disease, the gland may be enlarged due to inflammatory infiltrate, or small and atrophic, with fibrosis. Patients may show symptoms of an expanding intrasellar mass or different degrees of pituitary dysfunction. ${ }^{2}$

We report on a woman with lymphocytic hypophysitis presenting with clinical signs of acute hypopituitarism and idiopathic retroperitoneal fibrosis, probably of autoimmune origin. Lymphocytic hypophysitis should be included in the differential diagnosis of hypopituitarism at any age. The association with idiopathic retroperitoneal fibrosis suggests an autoimmune origin for this entity.

\section{Case report}

Department of Ciencias de la Salud I, University of la Coruña, La Coruña, Spain

F Cordido

Correspondence to Augusto Alvarez Garcia, Servicio de Anatomía Patológica, Hospital Juan Canalejo, Las Jubias 84, $15006 \mathrm{La}$ Coruña, España

Accepted 20 March 1997 A 78-year-old woman was admitted to the hospital because of right flank pain during the previous two weeks and renal failure. The patient had a 10-year history of bilateral obstructive uropathy with bilateral lithiasis and secondary renal atrophy. She was allergic to penicillin and had previous diagnoses of renal insufficiency and chronic anemia. She had never been pregnant. Physical examination disclosed general pallor, blood pressure of $130 / 70 \mathrm{mmHg}$ and body temperature of $37^{\circ} \mathrm{C}$. Cardiopulmonary auscultation showed a systolic murmur and bilateral rales. There was marked tenderness after percussion of the right costovertebral area. Laboratory blood tests revealed an erythrocyte count of $3.1 \times 10^{12} / 1$, haemoglobin $6.0 \mathrm{mmol} / 1$, haematocrit 0.267 , leucocyte count $8.7 \times 10^{9} / 1$, urea $34.1 \mathrm{mmol} / \mathrm{l}$, creatinine $335.9 \mathrm{mmol} / \mathrm{l}$, potassium $5.5 \mathrm{mmol} / \mathrm{l}$, sodium $135 \mathrm{mmol} / 1$ and glucose $6.1 \mathrm{mmol} / 1$. The urinary sediment presented abundant white blood cells and bacteria. Chest X-ray showed a right pleural effusion. Renal sonogram showed bilateral nephrolithiasis, high-grade right obstructive uropathy, and atrophic right kidney. The initial diagnosis was urinary tract infection complicating obstructive uropathy. Empiric antibacterial treatment was prescribed (aztreonam). Her condition worsened with vomiting, progressive decline in mental status and hypotension. Physical examination revealed blood pressure of $90 / 50 \mathrm{mmHg}$ and dehydration, without focal neurologic deficits. Repeated blood laboratory tests showed haemoglobin $5.6 \mathrm{mmol} / 1$, haematocrit 0.251 , leucocyte count $7.67 \times 10^{9} / 1$, urea $22.2 \mathrm{mmol} /$ 1, sodium $144 \mathrm{mmol} / \mathrm{l}$, potassium $5.7 \mathrm{mmol} / \mathrm{l}$, glucose $1.9 \mathrm{mmol} / 1$. Urine sodium was $49 \mathrm{mmol} / 1$ and urine potassium $57 \mathrm{mmol} / 1$. A presumptive diagnosis of acute adrenal insufficiency was established and the patient received high-dose hydrocortisone. Serum cortisol (before the steroid therapy) was $179 \mathrm{mmol} / \mathrm{l}$ (reference range $138-690 \mathrm{mmol} /$ 1). The thyroid-stimulating hormone level was $1.2 \mathrm{mU} / \mathrm{l}$ (reference range $0.25-4 \mathrm{mU} / \mathrm{l}$ ) and the free thyroxine was $7 \mathrm{pmol} / \mathrm{l}$ (reference range $11-24 \mathrm{pmol} / \mathrm{l})$. With these hormonal results and the highly stressful situation of the patient, a diagnosis of hypopituitarism was made. No stimulation test could be performed. A computed tomographic study did not show evidence of a hypothalamus-pituitary mass. After an initial clinical and biochemical improvement, with normalisation of serum glucose and electrolyte levels, the patient died seven days later due to bronchoaspiration.

An autopsy performed four hours after death

showed a normal brain $(1050 \mathrm{~g})$ and an atrophic pituitary gland, hard in consistency and white-grey in colour. The thyroid was small $(6.2 \mathrm{~g})$, as were the adrenal glands (joint weight $5 \mathrm{~g}$ ) and ovaries. There was a retroperitoneal fibrous mass with a diameter of $5.3 \mathrm{~cm}$, involving iliac vessels, both ureters and the right kidney hilium. Kidneys were atrophic, 
Figure 1 The posterior pituitary gland normal in size, showed no alterations (left), while the anterior part (right) is atrophic and by dense fibrous tissue (arrows) (HE original $\times 40$ )

Figure 2 Close view of the anterior pituitary gland with slight oedema and moderate lymphocytic infiltration surrounding (arrows) the residual parenchyma (HE original $\times 400$ )
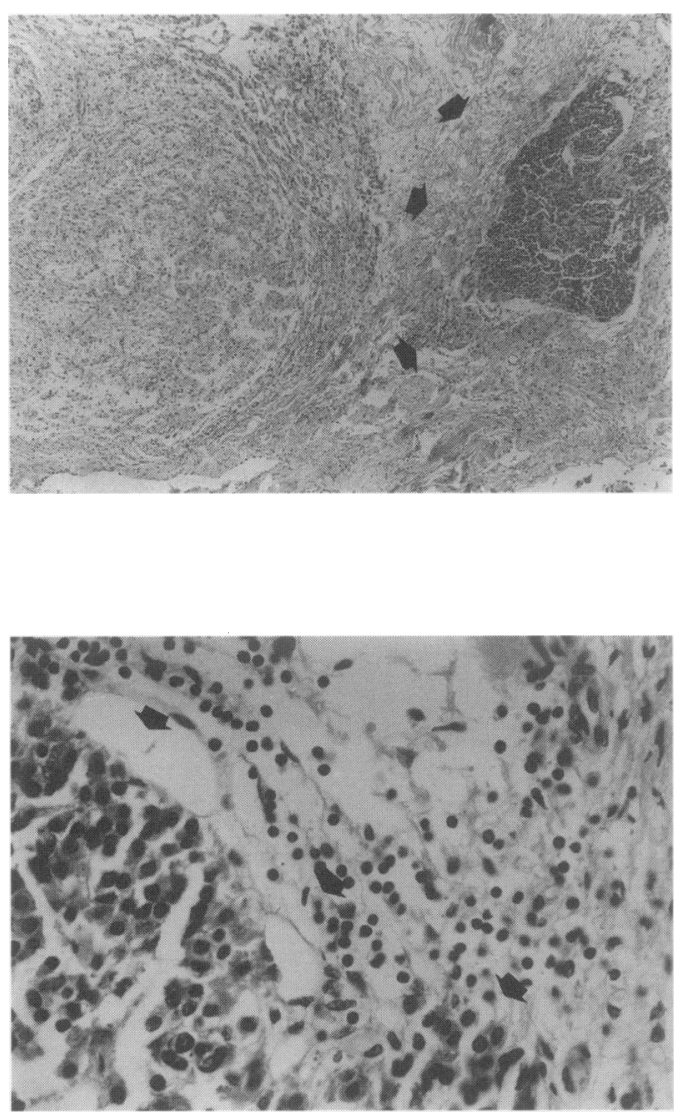

with pyelo-calyceal dilatation and coraliform lithiasis. The right kidney weighed $40 \mathrm{~g}$ and the left $130 \mathrm{~g}$. Other necropsy findings included an increase in the weight of both lungs $(600 \mathrm{~g}$ each) due to bilateral bronchopneumonia, and left ventricular hypertrophy due to subaortic stenosis.

Microscopically, the posterior pituitary gland was normal. Parenchymal fibrosis was found in the anterior pituitary gland (figure 1), with slight oedema and a moderate T-phenotype lymphocytic infiltration (CD3+), with plasmatic cells and few eosinophils (figure 2). No germinal centres, giant cells or granulomas were observed. The histologic appearance of the fibrous retroperitoneal mass was similar to that of the anterior pituitary gland, with collagen tissue and a scattered infiltrate of $T$ phenotype lymphocytes (CD3+) and polyclonal plasmatic cells. Isolated germinal centres were observed. Lymphocytic infiltrates and fibrosis were not observed in the thyroid, adrenal glands or ovaries.

\begin{tabular}{|l|}
\hline Causes of hypopituitarism \\
\hline - pituitary adenoma \\
- pituitary surgery \\
- pituitary radiation therapy \\
- empty sella syndrome \\
- head trauma \\
- infiltrative disease \\
- lymphornocytic hypophysitis \\
\hline
\end{tabular}

Causes of secondary retroperitoneal fibrosis

- drugs: methysergide, methyldopa, ergot alkaloids

- inflammation adjacent to an abdominal aneurysm

- malignant tumours

- retroperitoneal injuries: haemorrhage, infection, or radiation

- peri-arteritis nodosa

- autoimmune disease

- tuberculosis

- sarcoidosis

- biliary tract disease

- gonorrhoea

- ascending lymphangitis from the lower extremities

\begin{tabular}{|l|}
\hline Summary points \\
\hline - lymphocytic hypophysitis should be considered \\
a cause of hypopituitarism at any age \\
- it presents as an intrasellar mass during the last \\
months of pregnancy or early postpartum, or as \\
hypopituitarism at any age \\
- the pituitary can be enlarged or small in size \\
- the aetiology of lymphocytic hypophysitis is \\
probably autoimmune. Autoimmune \\
pathogenesis is supported by coexistence with \\
idiopathic retroperitoneal fibrosis \\
\hline
\end{tabular}

\section{Discussion}

Hypopituitarism without a pituitary tumour or previous pituitary-directed therapy could be due to pituitary apoplexy, empty sella syndrome, head trauma, internal carotid artery aneurysm and infiltrative diseases affecting the hypothalamus or the pituitary. ${ }^{1}$

Lymphocytic hypophysitis is a rare entity, with approximately 60 reported cases, predominantly affecting females and usually presenting during late pregnancy or the first 14 months thereafter. ${ }^{3}$ It presents as an intrasellar mass during the last three months of pregnancy or early postpartum period, or as postpartum hypopituitarism of variable degree. ${ }^{2}$ The loss of pituitary function is often out of proportion to the degree of pituitary enlargement. Depending on the phase of the disease, the pituitary gland can be enlarged, normal or small in size. Somatotropic and gonadotropic function are more likely to be preserved than corticotropic or thyrotropic function, unlike the findings in hypopituitarism due to a sizeable pituitary adenoma. ${ }^{2}$ It has been reported ${ }^{4}$ that antipituitary antibodies can be found in the serum of $75 \%$ of women with the empty sella syndrome. It is conceivable that in some cases the empty sella syndrome may represent a final stage of lymphocytic hypophysitis. ${ }^{2}$ In addition, in some cases of the empty sella syndrome, there is evidence of resolution of pituitary enlargement caused by previously unrecog- 
nised hypophysitis. ${ }^{3}$ The real incidence of this entity, therefore, may be greater than currently recognised. Some cases of idiopathic hypopituitarism in men and postmenopausal women may also be due to lymphocytic inflammation. Moreover, the spectrum of lymphocytic hypophysitis may include cases of idiopathic isolated adrenocorticotropin and TSH deficiencies. ${ }^{5,6}$

A definite diagnosis of lymphocytic hypophysitis requires histologic evidence of lymphoid inflammatory infiltrate involving the adenohypophysis, with plasmatic cells, eosinophils, fibrosis and oedema, without granuloma and/or epithelioid cells. ${ }^{2}$ Lymphocytic hypophysitis probably progresses through various stages. Early on, the pituitary becomes oedematous, inflamed, and enlarged, producing mass-like symptoms. Subsequently, the pituitary tissue is destroyed and replaced by fibrosis; the sellar mass spontaneously regresses, and symptoms of hypopituitarism develop. At this time the pituitary is usually shrunken and atrophic. The early stage may be subclinical, with no symptoms of pituitary mass but with ultimate development of postpartum hypopituitarism. ${ }^{2}$ The case here reported exemplifies the atrophic phase of lymphocytic hypophysitis with extensive fibrosis and scanty lymphoid infiltrate, features found only in late stages and diagnosed at autopsy. ${ }^{7}$

1 Vance ML. Hypopituitarism. N Engl f Med 1994; 330: $1651-63$.

2 Cosman F, Post KD, Holub DA, Wardlaw SL. Lymphocytic hypophysitis. Report of 3 new cases and review of the literature. Medicine 1989; 68: $240-56$.

3 Garber JR, Hedley-Whyte ET. Case 25-1995. A 44-year-old woman with headache, blurred vision, and an intrasellar mas. $N$ Engl f Med 1995; 333: $441-8$.

4 Komatsu M, Kondo T, Yamauchi K, et al. Antipituitary Komatsu M, Kondo T, Yamauchi K, et al. Antipituitary antibodies in patients with the primary empty

5 Burke CW, Moore RA, Rees LH, Bottazzo GF, Mashiter K, Bitensky L. Isolated ACTH deficiency and TSH deficiency in the adult. $\mathcal{F} R$ Soc Med 1979; 7: 328-35.

6 Stacpoole PW, Interlandi JW, Nicholson WE, Rabin D. Isolated ACTH deficiency: a heterogeneous disorder. Medicine 1982; 61: 13-24.
Because of the occasional presence of antipituitary antibodies, a lymphoid infiltration similar to that seen in autoimmune thyroiditis and the presence of activated lymphocytes interdigitated with pituicytes, the concept of lymphocytic hypophysitis as an autoimmune disease became firmly stabilised relatively early. ${ }^{4,8}$ In a third of the patients, lymphocytic hypophysitis is associated with other autoimmune diseases with similar histologic features: lymphoid thyroiditis, lymphoid adrenalitis, atrophic gastritis, parathyroiditis and idiopathic retroperitoneal fibrosis. ${ }^{7,9,10}$ On the other hand, its high incidence in pregnant or puerperal women favours an autoimmune aetiology due to the high risk of autoimmune exacerbations in these periods. The present case had an inflammatory infiltrate affecting the retroperitoneal fibrous tissue with similar immunologic phenotype and histologic features to the adenohypophysis tissue. These data suggest that both entities had a similar pathogenic mechanism.

In summary, lymphocytic hypophysitis should be included in the differential diagnosis of hypopituitarism, even if it appears in nonpregnant women or older patients. It is probably an autoimmune disease.

This work was supported by Xunta de Galicia Investigation Grant XUGA 90004 A-95.

7 Sobrinho-Simoes M, Brandao A, Paiva ME, Vilela B, Fernandes E, Carneiro-Chaves F. Lymphoid hypophysitis in a patient with lymphoid thyroiditis, lymphoid adrenalitis, and idiopathic retroperitoneal fibrosis. Arch Pathol Lab Med 1985; 109: $230-3$

8 Levine S. Allergic adenohypophysitis: a new experimental disease of the pituitary gland. Science 1967; 158: 1190-1.

9 Carpenter CCJ, Solomon N, Silverberg SG, et al. Schmidt's syndrome (thyroid and adrenal insufficiency): a review of syndrome (thyroid and adrenal insufficiency): a review of the literature and a report of fifteen cases including ten instances

10 Paja M, Estrada J, Ojeda A, Ramón y Cajal S, Garcia-Uria J, Lucas T. Lymphocytic hypophysitis causing hypopituitarism and diabetes insipidus, and associated autoimmune thyroiditis, in a non-pregnant woman. Postgrad Med $\mathcal{F} 1994$; 70: $220-4$. 